PESQUIMAT, Revista de la F. C. M. de la

Universidad Nacional Mayor de San Marcos

Vol. XI - N², pp. 71-80, Lima - Perú, Oct. 2008

\title{
DECAIMIENTO EXPONENCIAL DE UNA ECUACIÓN DE ONDA CON UNA CONDICIÓN DE FRONTERA VISCOELÁSTICA Y UN TERMINO FUENTE
}

\author{
V. Carrera B. ${ }^{1}$ \& F. León ${ }^{1}$ \& J. Bernui B. ${ }^{1}$ \& V. Martínez L. ${ }^{1}$
}

Resumen.- En este artículo estamos interesados en la estabilidad de las soluciones de una ecuación de onda con una condición de frontera viscoelástica y un término fuente, usaremos el método potencial, la técnica de multiplicadores y el teorema de unicidad para una ecuación de onda con coeficientes variables.

Palabras claves: Galerkin, decaimiento exponencial, ecuación de onda.

\section{EXPONENTIAL DECAY OF WAVE EQUATION WITH A VISCOELASTIC BOUNDARY CONDITION AND SOURCE TERM}

\begin{abstract}
In this paper we are concerned with the stability of solutions for the wave equation with a viscoelastic Boundary condition and source term by using the potential well method, the multiplier technique and unique continuation theorem for the wave equation with variable coefficient.
\end{abstract}

Key words: Galerkin, exponential decay, wave equation.

\section{Introduction}

The main purpose of this work is to study the asymptotic behavior of the solution of the following initial boundary problem.

$$
\begin{aligned}
& \left.u_{t t}-\left(a(x, t) u_{x}\right)_{x}=\mu|u|^{q-1} u \quad \text { in }\right] 0,1[x] 0,+\infty[ \\
& u(0, t)=0 \\
& u(1, t)+\int_{0}^{t} g(t-s) a(1, s) u_{x}(1, s) d s=0, \quad \forall t>0 \\
& u(x, 0)=u^{0}(x) \quad u_{t}(x, 0)=u^{1}(x)
\end{aligned}
$$

\footnotetext{
${ }^{1}$ UNMSMS, Facultad de Ciencias Matemáticas.
} 
The integral equation (1.3) is a Boundary condition with includes the memory effect. Here $u$ is the transverse displacement, $g$ the relaxation function and $\mu \in \mathbb{R}, q>1$. By $a=a(x, t)$ we represent a function of

$$
\begin{gathered}
W_{l o c}^{1, \infty}\left(0, \infty ; H^{1}(0,1)\right), \text { such that } a(x, t) \geq a_{0}>0, \\
\left.a_{t}(x, t) \leq 0 \text { and } a_{x}(x, t) \leq 0 \text { for all }(x, t) \in\right] 0,1[x] 0, \infty[
\end{gathered}
$$

There exist a large body of Literature Regarding viscoelastic problems with the memory term acting in the domain or in the Bondary. Among the numerous works in the directions we can cite Cavalcanti et al [2], Berrini \& Messaoudi [1], Messaoudi et al [3], Rivera et al [5], Santos [6], Park and Bae [4]. Considered the problem for the case of Kirchhoff type wave equation. All the authors mentioned above stablished thein results with $\mu \leq 0$.

The first part of this paper is to study the global existence of regular and weak solutions to problem $(1.1)-(1.4)$ when $\mu>0$; here we have some technical dificulties because of source term. Semigroup arguments are not suitable for finding solutions of $(1.1)-(1.4)$, the refore, we make use of Galerkin Aproximation and Potential well method.

The Second part is to give energy decay estimates of the solutions of (1.1) - (1.4); here the main difficulty is the source term, it seems that a straight forward adaption of method in [6] to our context fails completely. In order to solve this problem we need to introduce suitable multiplicadors and a unique continuation property for the wave equation with variable coefficients.

\section{Notation \& Preliminaries}

We denote

$$
(w, z)=\int_{0}^{1} w(x) z(x) d x, \quad|z|^{2}=\int_{0}^{1}|z(x)|^{2} d x
$$

By $V$ we denote the Hilbert Space

$$
V=\left\{w \in H^{1}(0,1): w(0)=0\right\}
$$

Denoting by

$$
(g * \varphi)(t)=\int_{0}^{t} g(t-s) \phi(s) d s
$$

the convolution product operador and differentiating (1.3) and the applying the Volterra's 
inverse operador, we get

$$
a(1, t) u_{x}(1, t)=-\frac{1}{g(0)}\left(u(1, t)+k * u_{t}(1, t)\right)
$$

where the resolvent kernel satisfies

$$
k(t)+\frac{1}{g(0)}\left(g^{\prime} * k\right)(t)=\frac{1}{g(0)} g^{\prime}(t)
$$

with $\tau=\frac{1}{g(0)}$ and using the above identity, we write

$$
a(1, t) u_{x}(1, t)=-\tau\left\{u_{t}(1, t)+k(0) u(1, t)-k(t) u_{0}(1)+k^{\prime} * u(1, t)\right\}
$$

Let us denote by,

$$
(f \square \varphi)(t)=\int_{0}^{1} f(t-s)\left|\varphi(t)-\varphi^{*}(s)\right|^{2} d s
$$

We introduce the following functionals:

$$
\begin{gathered}
J(t)=\frac{1}{2}\left|a^{1 / 2} u_{x}\right|^{2}-\frac{\mu}{q+1}|u|_{q+1}^{q+1} \\
E(t)=E\left(u(t), u_{t}(t)\right)=\frac{1}{2}\left|u_{t}\right|^{2}+J(t)+\frac{\tau}{2}\left(k(t)|u(1, t)|^{2}-k^{\prime}(t) \square u(1, t)\right) \\
I(t)=I(u(t))=a_{0}\left|u_{x}\right|^{2}-\mu|u|_{q+1}^{q+1}
\end{gathered}
$$

and define the stable set

$$
W=\left\{u \in V: I_{(u)}>0\right\} \cup\{\theta\}
$$

\section{Global Existence and Exponential Decay}

First, we need the following assumptions:

(A.1) The kernel $g$ is positive, and $k \in c^{2}\left(\mathbb{R}^{+}\right)$satisfies

$$
k,-k^{\prime}, k^{\prime \prime} \geq 0
$$

(A.2) Let us consider $\left\{u^{0}, u^{1}\right\} \in\left(H^{2}(0,1) \cap V\right) \times V$ verifying the compatibitity condition:

$$
a(1,0) u_{x}^{0}(1)=-\tau u_{1}(1)
$$


The we state our main result.

Theorem 3.1 Suppose that (A.1) - (A.2) hold;

$$
\mu>0,\left\{u^{0}, u^{1}\right\} \in\left(W \cap H^{2}(0,1)\right) \times V
$$

and

$$
\frac{\mu}{a_{0}} c_{*}^{q+1}\left[\frac{2(q+1)}{a_{0}(q-1)} E_{(0)}\right]^{\frac{(q-1)}{2}}<1
$$

Then there exists only $u$ of the system (1.1) - (1.4) satisfying

$$
\begin{gathered}
u \in L^{\infty}\left(0, \infty ; W \cap H^{2}(0,1)\right) \\
u_{t} \in L^{\infty}(0, \infty ; V) \\
u_{t t} \in L^{\infty}\left(0, \infty ; L^{2}(0,1)\right)
\end{gathered}
$$

Proof of theorem 3.1. The main idea is to use the Galerkin Method.

Let $\left\{w_{j}\right\}$ be a complete orthonormal system of $\mathrm{V}$ such that

$$
\left\{u^{0}, u^{1}\right\} \in \quad \operatorname{span}\left\{w^{0}, w^{1}\right\}
$$

and let us write

$$
u^{m}(t)=\sum_{j=1}^{m} h_{j m}(t) w^{j}
$$

where $u^{m}$ satisfies

$$
\begin{aligned}
\left(u_{t t}^{m}, w^{j}\right)+\left(a(x, t) u_{x}^{m}, w_{x}^{j}\right)= & \mu\left(\left|u^{m}\right|^{q-1} u^{m}, w_{j}\right) \\
& -\tau\left\{u_{t}^{m}(1, t)+k(0) u(1, t)+\right. \\
& \left.-k(t) u^{0}(1)+k^{\prime} * u(1, t)\right\} w^{j}(1)
\end{aligned}
$$

for $0 \leq j \leq m$, satisfying the following conditions

$$
u^{m}(0)=u^{0} \quad, \quad u_{t}^{m}(0)=u^{1}
$$

Standard results about ordinary differential equations guarantee that there exists only 
one solution of the system on some interval $\left[0, T_{m}[\right.$. The extension of the solution to the whole interval $[0, \infty[$ is a consequence of the first estimate which we are going to prove below.

Estimate I.- Multipliying (3.1) by $h_{j m}^{\prime}(t)$, integrating by parts and summing up on $j$ we get

$$
\begin{aligned}
\frac{d}{d t} E_{(t)}^{m}= & \left(a_{t} u_{x}^{m}, u_{x}^{m}\right) \\
& -\left\{\tau\left|u_{t}^{m}(1, t)\right|^{2}+k(0) u^{m}(1, t) u_{t}^{m}(1, t)+\right. \\
& \left.-k(t) u^{0}(1) u_{t}(1, t)+k^{\prime} * u^{m}(1, t) u_{t}(1, t)\right\}
\end{aligned}
$$

where $E^{m}(t)=E\left(u^{m}(t)\right)$.

Moreover, we know that for $f, \varphi \in C^{1}([0, \infty[, \mathbb{R})$ we have

$$
\begin{aligned}
\int_{0}^{t} f(t-s) \varphi(s) d s \varphi_{t}= & -\frac{1}{2} f(t)|\varphi(t)|^{2}+\frac{1}{2} f^{\prime} \square \varphi \\
& -\frac{1}{2} \frac{d}{d t}\left[f \square \varphi-\left(\int_{0}^{t} f(s, d s)|\varphi|^{2}\right)\right]
\end{aligned}
$$

Applying (3.3) to the term $k^{\prime} * u^{m}(1, t) u_{t}^{m}(1, t)$ in (3.2) and using the properties of $k, k^{\prime}$ and $k^{\prime \prime}$ we conclude by (3.2)

$$
\frac{d}{d t} E^{m}(t) \leq c E^{m}(0)
$$

Taking into account the definition of the initial data of $u^{m}$ we conclude that

$$
E^{m}(t) \leq c, \forall t \in[0, T], \forall m \in \mathbb{N}
$$

Lema 3.2 Let $u^{m}(t)$ be the solution of (3.1) with $u^{0} \in W$ and $u^{1} \in L^{2}(0,1)$.

If

$$
\frac{\mu}{a_{0}} c_{*}^{q+1}\left[\frac{2(q+1)}{a_{0}(q-1)} E(0)\right]^{\frac{q-1}{2}}<1
$$

then $u^{m}(t) \in W$ on $[0, T]$; that is, for all $t \in[0, T]$

$$
I\left(u^{m}(t)\right)>0
$$


Proof.- Since $I\left(u^{0}\right)>0$, it follows from the continuity of $u^{m}(t)$ that

$$
I\left(u^{m}(t)\right) \geq 0 \text { for some interval near to } t=0
$$

Let $t_{\max }>0$ be a maximal time (possibly $t_{\max }=T_{m}$ ) such that (3.4) holds on $\left[0, t_{\max }[\right.$

In order to facilitate the notation, we will omite the index $m$ of the approximate system. Note that

$$
\begin{aligned}
J(u(t)) & =\frac{1}{2}\left(a u_{x}, u_{x}\right)-\frac{\mu}{q+1}|u|_{q+1}^{q+1} \geq \frac{a_{0}}{2}\left|u_{x}\right|^{2}-\frac{\mu}{q+1}|u|_{q+1}^{q+1} \\
& =\frac{1}{q+1} I(u)+\frac{a_{0}(q-1)}{2(q+1)}\left|u_{x}\right|^{2} \geq \frac{a_{0}(q-1)}{2(q+1)^{\cdot}}\left|u_{x}\right|^{2} \\
\forall t & \in\left[0, t_{\max }[\right.
\end{aligned}
$$

Consequently, we get

$$
\left|u_{x}\right|^{2} \leq \frac{2(q+1)}{a_{0}(q-1)} J(u) \leq \frac{2(q+1)}{a_{0}(q-1)} E(t) \leq \frac{2(q+1)}{a_{0}(q-1)} E(0)
$$

It follows from the Sobolev-Poincaré inequality and (3.5) that

$$
\begin{aligned}
\mu|u|_{q+1}^{q+1} & \leq \mu c_{*}^{q+1}\left|u_{x}\right|^{q+1} \leq \frac{\mu c_{*}^{q-1}}{a_{0}}\left|u_{x}\right|^{q+1}\left(a_{0}\left|u_{x}\right|^{2}\right) \\
& \leq \frac{\mu c_{*}^{q+1}}{a_{0}}\left[\frac{2(q+1)}{a_{0}(q-1)} E(0)\right]^{\frac{q-1}{2}}\left(a_{0}\left|u_{x}\right|^{2}\right)<a_{0}\left|u_{x}\right|^{2}
\end{aligned}
$$

This, from (3.6) obtain

$$
\mu|u|_{q+1}^{q+1}<a_{0}\left|u_{x}\right|^{2}
$$

Therefore we get $I(u)>0$ on $\left[0, t_{\max }\left[\right.\right.$. This implies that we can take $t_{\max }=T_{m}$. This completes the proof of lemma.

Remark 1 Let $u$ be as in lemma 3.2, then there is a certain number $n_{0}, \quad 0<n_{0}<1$ such that

$$
\mu|u|_{q+1}^{q+1} \leq\left(1-n_{0}\right)\left(a u_{x}, u_{x}\right)
$$


In fact, from lemma 3.2

$$
\mu|u|_{q+1}^{q+1} \leq\left(1-n_{0}\right) a_{0}\left|u_{x}\right|^{2} \leq
$$

with $n_{0}=1-\frac{\mu c_{x}^{q+1}}{a_{0}}\left[\frac{2(q+1)}{a_{0}(q-1)} E(0)\right]^{\frac{q-1}{2}}$.

Using lemma 3.2 we can deduce a priori estimate for $u^{m}(t)$. Lemma 3.2 implies that

$$
\begin{aligned}
E(t)=\frac{1}{2}\left|u_{t}\right|^{2}+\frac{\tau}{2}\left(k(t)|u(1, t)|^{2}-k^{\prime}(t) \square u(1, t)\right)+J(u) \\
\geq \frac{1}{2}\left|u_{t}\right|^{2}+\frac{a_{0}(q-1)}{2(q+1)}\left|u_{x}\right|^{2}+\frac{1}{q+1} I(u) \\
\geq \frac{1}{2}\left|u_{t}\right|^{2}+\frac{a_{0}(q-1)}{2(q+1)}\left|u_{x}\right|^{2}
\end{aligned}
$$

Then

$$
\frac{1}{2}\left|u_{x}\right|^{2}+\frac{a_{0}(q-1)}{2(q+1)}\left|u_{x}\right|^{2} \leq E(t) \leq E(0) \leq 1
$$

Where $L_{1}$ is a positive constant independent of $m \in \mathbb{N}$ and $t \in[0, T]$.

Estimate II.- Next, we shall find a estimate for the second order energy. Fisrt, let us estimate the initial data $u_{t t}^{m}(0)$ in the $L^{2}-$ norm. Letting $t \rightarrow 0^{+}$in the equation (3.1), multiplying the result by $h_{j m}(0)$ and using the compatibility condition we get

$$
\left|u_{t t}^{m}(0)\right| \leq M_{1}, \quad \forall m \in \mathbb{N}
$$

Differentiating (3.1) with respect to the time, multiplying by $h_{j m}^{\prime \prime}(t)$ and summing us the products results in $j$, noting that

$$
\left|\mu\left(|u|^{q-1} u_{t}, u_{t t}\right)\right| \leq\left|u_{x}\right|^{q-1}\left|u_{x t}\right|\left|u_{t t}\right|
$$


after some computations we obtain

$$
\begin{aligned}
\frac{d}{d t} E_{1}^{m}(t) \leq & \frac{1}{2}\left(a_{t} u_{x_{t}}, u_{x_{t}}\right)-\frac{\tau}{2}\left|u_{t t}(1, t)\right|^{2} \\
& +\frac{1}{4 \eta}\left|k^{\prime}(t)^{2} \| u^{0}(1)\right|^{2}+\frac{1}{4 \eta}\left|k^{\prime}(0)\right|^{2}|u(1, t)|^{2}+ \\
& +\frac{1}{4 \eta}\left|k^{\prime \prime}\right|_{L^{\prime}(0, \infty)}\left|k^{\prime \prime}\right| \square u(1, t)+c\left(\left|u_{x t}\right|^{2}+\left|u_{t t}\right|^{2}\right)
\end{aligned}
$$

for some $n, c>0$, where

$$
E_{1}^{m}(t)=\frac{1}{2}\left|u_{t t}\right|^{2}+\frac{1}{2}\left(a u_{x t} \cdot u_{x t}\right)+\frac{1}{2} k(0)\left|u_{t}(1, t)\right|^{2}
$$

By integrating (3.7) over $[0, t]$ and employing the first estimate and Gronwall's lemma we obtain

$$
E_{1}^{m}(t) \leq c \quad, \quad \forall t \in[0, T], \forall m \in \mathbb{N}
$$

the rest of the proof is a matter of routine.

Proof. To Prove this theorem we shall use the Galerkin Method and potential well theory for the wave equation.

\section{Uniform Decay}

\subsection{Exponential Decay}

In this section, we shall show the asymptotic behavior of solutions for system (1.1) (1.4) when the resolvent kernel $k$ decay exponentially, that is, there exist positive constants $m_{1}, m_{2}$ such that

$$
k(0)>0 \quad ; \quad k^{\prime}(t) \leq-m_{1} k(t) \quad ; \quad k^{\prime \prime}(t) \geq-m_{2} k^{\prime}(t)
$$

Note that this implies that

$$
k(t) \leq k(0) e^{-m_{1} t}
$$

At first, we begin with the following Lemmas. 
Lemma 4.1 Any strong solution of system (1.1) - (1.4) satisfies

$$
\begin{aligned}
\frac{d}{d t} E(t) \leq & -\frac{\tau}{2}\left|u_{t}(1, t)\right|^{2}+\frac{\tau}{2} k^{2}(t)\left|u_{0}(1)\right|^{2}+\frac{\tau}{2} k^{\prime}(t)|u(1, t)|^{2}+ \\
& -\frac{\tau}{2} k^{\prime \prime}(t) \square u(1, t)+\frac{1}{2}\left(a_{t} u_{x}, u_{x}\right)
\end{aligned}
$$

Proof. Multiplying (1.1) by $u_{t}$ and integrating over [0,1], our conclusion follows.

As a consequence of the above Lemma we have that energy is bounded for any $t \geq 0$.

Lemma 4.2 Any stong solution of system (1.1) - (1.4) satisfies

$$
\begin{aligned}
\frac{d}{d t}\left(u_{t}, x u_{x}\right) \leq & \left(\frac{1}{2}+\frac{\tau^{2}}{2 \varepsilon}\right)\left|u_{t}(1, t)\right|^{2}+\frac{|k(t)|}{\varepsilon}\left|k^{\prime}\right| \square u(1, t)+\frac{|k(0)|^{2}}{\varepsilon}|u(1, t)|^{2}+ \\
& +\frac{1}{2 \varepsilon} k(t)^{2}\left|u_{0}(1)\right|^{2}+2 \varepsilon\left|u_{x}(1, t)\right|^{2}-\frac{\alpha}{4} E(t)+c|u|^{2}
\end{aligned}
$$

Proof. Multiplying Equation (1.1) by $x u_{x}$, using the Boundary condition (1.7) taking $\varepsilon$ small enough, we arrive at the conclusion.

Lema 4.3 Let $u$ be a solution in theorem 3.1. Suppose that (4.1) holds and the initial data verifies

$$
u^{0}(1)=u_{x}^{0}(1)=0
$$

The there exists $T_{0}>0$ such that if $T \geq T_{0}$ we have

$$
\int_{S}^{T}|u|^{2} d s \leq c \int_{S}^{T}\left|u_{t}(1, s)\right|^{2} d s
$$

where $c$ is $A$ positive constant.

Proof.-The method we use is based on A compactness-Uniqueness argument. In order apply this method we need an unique continuation theorem for the wave equation with variable coefficients. Let us introduce the functional

$$
L(t)=N E(t)+\left(u_{t}, x u_{x}\right)
$$


with $N>0$. Using Young's inequality and taking $\mathrm{N}$ large enough we find that

$$
\theta_{0} E(t) \leq L(t) \leq \theta_{1} E(t)
$$

For some positive constants $\theta_{0}$ and $\theta_{1}$.

Applying Lemmas 4.1-4.3, and integrating from $\mathrm{s}$ to $\mathrm{t}$ where

$$
0 \leq s \leq T<+\infty
$$

we obtain

$$
\int_{S}^{T} E(t) d t \leq c E(s)
$$

this condition implies that

$$
E(t) \leq M E(0) e^{-\gamma t}
$$

wich completes the Proof.

\section{REFERENCES}

[1] S. Berrini, S.A. Messaoudi - Existence and Decay of solutions of A Viscoelastic Equation with A nonlinear source. Nonlinear Anal, T.M.A. 64 (2006) 23/4-2331.

[2] M. M. Cavalcanti, V. N. Domingos Cavalcanti, J.S. Prates Filho, and J. A. Soriano - Existence and Uniform Decay for Viscoelastic Problems with nonlinear Boundary Damping. Diff. Integ. Eqs. 14 (2001), pp. 85-116.

[3] S.A. Messaoudi; and A. Soufyane - Boundary Stabilization of solutions of a nonlinear system of Timoshenko type. Nonlinear. Anal, T.M.A. 64 (2007), pp. 2107-2121.

[4] J. Y. Park, J. J. Bae - On coupled wave equation of Kirchhoff type with nonlinear Boundary Damping and memory terms. Appl. Math. Comp 129 (2002), pp. 87-105.

[5] J. E. Muñoz Rivera, D, Andrade - Exponential Decay of Nonlinear wave Equation with a viscoelastic Boundary condition. Math Meth. Appl. Sci, 23, pp. 41-61 (2000).

[6] M. L. Santos - Decay Rates for Solutions of semilinear wave equations with a memory condition at the Bondary, EJQTDE Nº7 (2002), pp. 1-17. 